\title{
Cytokine Production and Treatment Response in Major Depressive Disorder
}

\author{
S. Lanquillon, M.D., J.-C. Krieg, M.D., U. Bening-Abu-Shach, and H. Vedder, M.D.
}

In a controlled study, such immunological parameters as whole blood production of the cytokines interleukin-6 (IL-6) and tumor-necrosis factor- $\alpha$ (TNF- $\alpha$ ) were assessed in 24 inpatients with a major depressive disorder (MDD) both before and again under treatment. After a 6-week treatment period with amitriptyline, patients were classified as responders or nonresponders according to their psychopathological outcome as evaluated by the Hamilton and the Montgomery-Asberg Depression Rating Scales. Pre-treatment levels of c-reactive protein (CRP) were significantly higher in both patient subgroups than in the control subjects. In comparison to the controls, unstimulated pretreatment production of IL-6 was significantly decreased in the responders; whereas it was significantly increased in the nonresponder subgroup. Post- treatment values did not differ significantly among the patient and control groups. Pretreatment levels of TNF- $\alpha$ were increased in both patient subgroups, with a significant decrease during treatment only in the responder subgroup. Pretreatment levels of IL-6/10 mononuclear cells and the ratio between lymphocytes and monocytes acted as independent variables with regard to the clinical response. Our data indicate that unstimulated secretion of TNF- $\alpha$ is related to the psychopathological improvement; whereas, IL-6 levels might dichotomize the patients into subsequent responders and nonresponders already at admission.

[Neuropsychopharmacology 22:370-379, 2000] (C) 2000 American College of Neuropsychopharmacology. Published by Elsevier Science Inc.
KEY WORDS: Major depression; Immune system;

Interleukin-6; Tumor-necrosis factor- $\alpha$; Treatment response

Increasing data demonstrate changes of immune parameters in different psychiatric illnesses, including major depressive disorder (MDD) (for review see: Connor and Leonard 1998; Maes 1995; Müller and Ackenheil 1998). Alterations include increased concentrations of a number of cytokines, such as interleukin-6 (IL-6), interleukin-1 $\beta$ (IL-1 $\beta$ ), and interferon- $\gamma$ (INF- $\gamma)$. Moreover, changes in the concentrations of some of the re-

From the Department of Psychiatry and Psychotherapy, PhilippsUniversity of Marburg, Marburg, Germany.

Address correspondence to: Dr. H. Vedder, Department of Psychiatry and Psychotherapy, Philipps-University of Marburg, RudolfBultmann-Str. 8 D-35033 Marburg, Germany.

Received April 5, 1999; revised September 27, 1999; accepted September 30, 1999. spective receptors, such as the IL-2 and the IL-6 receptor (Maes et al. 1991; Maes et al. 1993; Maes 1995) have been detected. Other results indicate numerical (Maes et al. 1992; Ravindran et al. 1998; Seidel et al. 1996) and functional alterations of cellular immune parameters, such as neutrophil and monocyte phagocytosis (McAdams 1993). These data point to cellular aberrations as a possible cause of the secretory changes in the cytokine and other systems, such as parameters of the acute phase response.

IL-6 has been examined under several experimental conditions in depressed patients. The majority of the results showed an increase in the blood levels of this cytokine in these patients (Berk et al. 1997; Frommberger et al. 1997; Maes et al. 1994; Maes et al. 1995a; Maes et al. 1995b; Maes et al. 1997; Seidel et al. 1995; Sluzewska et al. 1996). Despite the important effects of the cytokine TNF- $\alpha$ on behavioral, endocrine, and immune parame- 
ters in the rat (Connor et al. 1998), the database on this cytokine in depressed patients is poor. To our knowledge, only one study examined the levels of TNF- $\alpha$ in elderly women with chronic MDD and found no alterations of this cytokine (Brambilla and Maggioni 1998). In addition to these cytokines, other immune parameters, including $\alpha 1$-glycoprotein (AGP), have been investigated with regard to psychopathological improvement and antidepressive treatment (Frommberger et al. 1997; Maes et al. 1995b; McAdams 1993; Seidel et al.1996; Sluzewska et al. 1995b; Sluzewska et al. 1996; Weizman et al. 1994). The association of some of these factors (IL-6, soluble IL-6 receptor, AGP, IL-1 receptor antagonist, IL-1 $\beta$ ) with the depressive symptomatology points to their possible state marker character as suggested by Maes et al. (1995a) and Connor and Leonard (1998). These results also show an immune activation during the acute stage of the disease. Other studies suggest that the severity of the disease and, hence, the exactness of the psychopathological assessment are important factors that may affect the subsequent examination of immunological functions (Brambilla and Maggioni 1998; Maes et al. 1995b; Weizman et al. 1994).

In the present controlled study, we examined immunological parameters, including the cytokines IL- 6 and TNF- $\alpha$, in the blood of depressed inpatients with regard to the treatment response. The main hypothesis was that the immunological status in MDD is related to the psychopathological course of the disease. For treatment, amitriptyline was used because of its variety of effects on multiple neurotransmitter systems. After a 6week treatment period, patients were classified as responders or nonresponders, and their immunological and psychopathological parameters were then compared with the data obtained at admission and with those of age-matched normal controls.

\section{METHODS}

\section{Characterization of Patients and Controls}

Depressed patients, consecutively admitted for inpatient treatment, were screened for fulfilling the DSM-IV diagnostic criteria (American Psychiatric Association 1994) for "major depressive disorder, single episode" (296.2) or "major depressive disorder, recurrent" (296.3). The clinical diagnosis was confirmed by the Structured Clinical Interview for DSM-IV (SCID, German Version; Wittchen et al. 1995). Patients had to be free of antidepressive medication for at least 6 weeks or of neuroleptics for at least 6 months before hospital admission. Moreover, serum and urine screens for antidepressants and substances typical of drug abuse had to be negative. Further exclusion criteria were any additional axis I or axis II DSM-IV diagnosis, pregnancy, acute or chronic infectious, autoimmune, allergic, neoplastic, or endocrine diseases and other acute physical diseases, including surgery or infarction of the heart or brain within the last 3 months. These illnesses were ruled out by clinical interview, physical examination, and comprehensive laboratory work-up focusing on parameters indicative of inflammation.

Age- and sex-matched healthy volunteers with no lifetime or current diagnosis of any psychiatric disorder served as control subjects and underwent the same diagnostic procedures as the depressed patients to rule out any of the above- mentioned exclusion criteria.

In the depressed patients, the psychopathology was quantified by the HAMD (Hamilton Depression Rating Scale; 21-item version) (Hamilton 1960) and the MADRS (Montgomery-Asberg Depression Rating Scale) (Montgomery and Asberg 1979) at admission and after 2, 3, 4, 5, and 6 weeks of inpatient treatment. Although the scores of the HAMD scale are known to correlate significantly with those of the MADRS, we decided to use both scales, because the HAMD more extensively covers somatic and psychomotor symptoms than the MADRS, which in turn, is alleged to be particularly sensitive when measuring treatment effects. Clinical and laboratory data were anonymous, and all ratings were performed by a single experienced psychiatrist who was blind to the immunological results. Amitriptyline was used as antidepressant throughout the study and increased in steps of $25 \mathrm{mg}$ at every second day, until efficient individual dosages between 150 and $250 \mathrm{mg} /$ day were reached according to clinical judgment. Plasma levels were measured after the final dosage was given for several days. As concomitant medication, treatment with lorazepam (1-5 mg/day) was allowed for the first 2-3 weeks of treatment. Longstanding medication for such mild chronic diseases (see also exclusion criteria) as heart disease was continued as long as it did not interfere with immune processes. Immunological parameters were assessed before treatment and at the end of the study. The endpoints were set either at a minimum of $50 \%$ reduction of the initial HAMD- and MADRS-scores lasting at least for a 1week period (clinical remission $=$ responder) or after 6 weeks with a reduction below $50 \%$ (not sufficiently remitted $=$ nonresponder).

Overall, 35 inpatients entered the study. Of these, 11 dropped out for various reasons, including new signs of acute physical disease (3), substance abuse (3), histrionic personality disorder (2), and dismissal for other reasons (3) before remission or before the prospected time period of 6 weeks was over. Twenty-four patients completed the study and were matched with 15 controls. The study protocol was approved by the ethics committee of the Philipps-University of Marburg and written informed consent was obtained from all participants. 


\section{Immunological Parameters}

Standard immunological parameters (erythrocyte sedimentation rate, c-reactive protein [CRP], differential blood cell counts) were determined according to routine methods. Differential blood cell counts of lymphocytes and monocytes (taken together as peripheral mononuclear cells [PMNCs]) were evaluated microscopically after Giemsa stain and expressed as percentages of white blood cell counts determined automatically (Cell Dyn 1700; Abott, Frankfurt, Germany). All cell numbers were expressed as cells $/ \mu$ l blood.

For detection of cytokines, adapted commercially available tests (Biosource, Ratingen, Germany) for the determination of whole blood-derived IL- 6 and TNF- $\alpha$ were employed (DeGroote et al. 1993). Whole blood was used to exclude a possible interference with external factors, such as purification procedures or artificial stimulation. Briefly, out of $5 \mathrm{ml}$ blood, collected into pyrogen-free heparinized syringes at $8.00 \mathrm{AM}, 25 \mu \mathrm{l}$ of whole blood were added to $200 \mu \mathrm{l}$ of Roswell Park Memorial Institute (RPMI) medium (Biosource) in antibody-coated microtiter-wells. Supplied standards were used for the standard curve. Plates were incubated for $24 \mathrm{~h}$ (IL-6) or $3 \mathrm{~h}$ (TNF- $\alpha$ ) at $37^{\circ} \mathrm{C}$, washed eight times, and incubated with $150 \mu \mathrm{l}$ (IL-6) or $200 \mu \mathrm{l}$ (TNF- $\alpha$ ) of secondary antibody solution (for IL-6: with a desensitizer solution). Detection was achieved with horseradish-peroxidase, $100 \mu \mathrm{l}$ chromogen solution (tetramethylbenzidine) and determination of the optical density (OD) at $450 \mathrm{~nm}$ in a microplate reader (Dynex, Nürtingen, Germany). Concentrations in the samples were calculated from a linear reference curve derived from the provided standards and expressed as pg cytokine $/ \mathrm{ml}$ blood volume. Intra-assay coefficients of variance $(\mathrm{CV})$ with the blood samples were determined to be less than $10 \%$ for both cytokines and different concentrations within the range of the standard curve, except for the $250 \mathrm{pg} / \mathrm{ml}$ concentration of IL-6 (11.2\%). Interassay CV were less than $9 \%$ for IL-6, and less than $8 \%$ for TNF- $\alpha$.

\section{Data Analysis}

Immunological parameters were assessed once for the controls and twice for the patients as pre- and posttreatment values. Patients were divided into responders and nonresponders according to the psychopathological criteria described above, and data were compared among the three groups.

The production of cytokines was expressed as cytokines released per $10^{5} \mathrm{PMNCs}$, if not mentioned otherwise. Further evaluation of immunological data included the calculation of ratios between the numbers of the different blood cells. Finally, data were additionally analyzed for correlations between the amounts of lym- phocytes and monocytes, cytokine production, and the treatment response.

Because most data were not strictly normally distributed and some groups had unequal variances, nonparametric tests were used for the evaluation of most results: the Kruskal-Wallis analysis of variance (ANOVA) on ranks for comparisons between patient subgroups and controls and the Mann-Whitney test for comparisons between patients and controls. For post hoc comparisons, Dunn's test was applied. The Wilcoxon matchedpairs test was used for the analysis of differences within a patient subgroup between two time points. Correlation testing between continuous variables was performed by the Pearson coefficient for linear regression. The Spearman correlation analysis on ranks was used if the regression was nonlinear. To estimate which continuous variables (immunological parameters) had the highest predictive value for the reduction of psychopathometric scores in the patients, stepwise multiple regression analysis was carried out. The $\alpha$-level for significance was set at 0.05 in all tests. For all analyses, STATISTICA 5.0 (StatSoft, OK) was used as statistical software.

\section{RESULTS}

\section{Clinical Data}

The demographic and psychometric data of the subjects participating in the study are shown in Table 1A. $H A M D$ - and MADRS-scores did not differ between patients who completed the study and those who dropped out, which makes an interfering bias because of exclusion from the study unlikely (Table 1A). Under the treatment condition, the HAMD (MADRS) scores decreased from $27.9 \pm 3.7(29.2 \pm 3.1)$ to $7.8 \pm 4.0(9.1 \pm$ 4.1) in the responders $(n=14)$ and from $27.5 \pm 5.0(31.3 \pm$ $5.5)$ to $18.9 \pm 5.0(23.2 \pm 5.4)$ in the nonresponders $(n=$ 10). The pretreatment scores of the responders did not differ from those of the nonresponders (HAMD: MannWhitney test, $p=.62$, NS; MADRS: Mann-Whitney test, $p=.43$, NS) (Table 1B). Neither the number of previous depressive episodes (Mann-Whitney test, $p=.73$, NS) nor the duration of the current episode (Mann-Whitney test, $p=$ .64, NS) affected the clinical outcome. Final plasma levels of the antidepressant were slightly, but not significantly, lower in responders $(369 \pm 112 \mu \mathrm{g} / \mathrm{l})$ than in nonresponders $(408 \pm 133 \mu \mathrm{g} / \mathrm{l})$ (Mann-Whitney test, $p=$ $.59, \mathrm{NS})$.

\section{Immunological Data}

The standard immunological parameters of lymphocyte and monocyte cell numbers showed no differences between controls and patient subgroups (for lymphocytes: 
Table 1. (A) Demographic and Psychopathometric Data of Patients with MDD and Healthy Controls. (B) Psychopatometric Data Grouped According to the Treatment Response

\begin{tabular}{|c|c|c|c|c|c|}
\hline \multicolumn{3}{|l|}{$\mathbf{A}^{a}$} & Patients in study & Drop-outs & Controls \\
\hline \multicolumn{3}{|c|}{ No. of subjects } & 24 & 11 & 15 \\
\hline \multicolumn{3}{|c|}{ female/male } & $15 / 9$ & $7 / 4$ & $9 / 6$ \\
\hline \multicolumn{3}{|c|}{ Age (years) } & $53.5(15.2)$ & $48.7(14.1)$ & $52.3(13.5)$ \\
\hline \multicolumn{3}{|c|}{ HAMD - Pre-treatment (score) } & $26.5(4.3)$ & $25.8(5.2)$ & $0.9(1.1)$ \\
\hline \multicolumn{3}{|c|}{ HAMD - Post-treatment (score) } & $9.4(6.8)$ & $8.9(5.4)$ & - \\
\hline \multicolumn{3}{|c|}{ MADRS - Pre-treatment (score) } & $27.8(3.5)$ & $27.2(3.1)$ & $0.8(1.1)$ \\
\hline \multicolumn{3}{|c|}{ MADRS - Post-treatment (score) } & $11.6(8.1)$ & $10.4(7.8)$ & - \\
\hline \multicolumn{3}{|c|}{ Number of episodes (n) } & $2.8(0.43)$ & $2.3(0.72)$ & \\
\hline \multicolumn{3}{|c|}{ Duration of current episode (months) } & $2.9(2.7)$ & $3.2(2.9)$ & \\
\hline \multicolumn{3}{|c|}{ Patients with weight loss (n) } & $10 / 24$ & & \\
\hline \multicolumn{3}{|l|}{ B } & Pre-treatment & Post-treatment & $\%$ Reduction \\
\hline \multirow{3}{*}{ HAMD } & & $\mathrm{N}$ & Mean (SD) & Mean (SD) & Mean \\
\hline & -Responders & 14 & $27.9(3.7)^{b}$ & $7.8(4.0)^{b}$ & 72.0 \\
\hline & -Non-Responders & 10 & $27.5(5.0)^{c}$ & $18.9\left(5.0^{c}\right.$ & 31.6 \\
\hline \multirow[t]{2}{*}{ MADRS } & -Responders & 14 & $29.2(3.1)^{b}$ & $9.1(4.1)^{b}$ & 68.8 \\
\hline & -Non-Responders & 10 & $31.3(5.5)^{c}$ & $23.2(5.4)^{c}$ & 26.1 \\
\hline
\end{tabular}

\footnotetext{
${ }^{a}$ Age, HAMD, MADRS, Number of episodes, Duration ... : : Mean (SD)

${ }^{b} p<.001$ (Wilcoxon-test).

${ }^{c} p<.01$ (Wilcoxon-test).
}

ANOVA; $p=.24, \mathrm{H}=2.89$; $\mathrm{df}=2$ ) (for monocytes: ANOVA; $p=.07, \mathrm{H}=5.43$; $\mathrm{df}=2$ ) (Table 2A). At admission, absolute monocyte counts were increased in the responder subgroup, although not to a significant extent, and normalized after therapy (Table 2A). The ratio of lymphocytes/monocytes before treatment was significantly lower in responders than in controls (ANOVA; $p<$ $.05, \mathrm{H}=6.27 ; \mathrm{df}=2$ ) (Dunn's test; $p<.05$ ) and higher in nonresponders, although not to a significant extent. In both groups of patients, values nearly normalized after treatment (Table 2B). Levels of c-reactive protein $(C R P)$ were significantly increased in patients at admission (ANOVA; $p<.001 ; \mathrm{H}=15.21 ; \mathrm{df}=2$ ) and decreased in both responders and nonresponders during treatment (Wilcoxon tests: $p<.05$ ) without significant differences between the two groups (Table 2C). The erythrocyte sedimentation rate (ESR) did not show any differences among controls, responders, and nonresponders (ANOVA; $p=.15, \mathrm{H}=6.27 ; \mathrm{df}=2$ ) (Table 2C).

The correlation of cell numbers with the reduction of psychopathometric scores revealed a weak, not yet significant positive correlation only for monocytes (Pearson correlation coefficient; $\mathrm{r}=0.30 ; p=.07$ ); whereas, the pretreatment ratio of lymphocytes/monocytes significantly correlated negatively with the reduced posttreatment scores in psychopathology (Pearson correlation coefficient; $\mathrm{r}=-0.43 ; p<.01$ ) (Table $3 \mathrm{~B}$ ).

There was no statistical difference in the production of $I L-6$ between patients and controls, although patients had slightly higher values at admission (Mann-Whitney test; $p=.95$, NS) (Figure 1A). After dividing patients into responders and nonresponders, statistical analysis revealed significant differences between the groups (ANOVA; $p<.0001 ; \mathrm{H}=16.9$; $\mathrm{df}=2$ ). Patients with subsequent remission showed significantly lower IL-6 values at admission than controls (Dunn's test; $p<$ .05 ) or patients without remission (Dunn's test; $p<.05$ ) (Figure 1B). Nonremitting patients produced significantly higher IL-6 levels as compared to the controls (Dunn's test; $p<.05$ ) and to responders before treatment (Dunn's test; $p<.05$ ). Regardless of the clinical outcome, IL-6 secretion returned to control levels after treatment in the responder (Wilcoxon test; $p<.01$ ) and in the nonresponder subgroup, respectively (Wilcoxon test; $p<.05$ ) (Figure 1B). In nonresponders, there was a significant positive hyperbolic correlation between monocyte numbers and IL-6 production at admission (Spearman Rank correlation coefficient; $\mathrm{r}_{\mathrm{S}}=0.88 ; p<$ .01) (Table 3A, Figure 2). A significant negative correlation was found between the degree of clinical improvement and the pretreatment levels of unstimulated IL-6 at admission (Pearson correlation coefficient; $\mathrm{r}=-0.62$; $p<.01$ ) (Table 3B). In contrast to the responders, higher degrees of clinical severity of MDD, as quantified by the MADRS score, at admission were negatively correlated with pretreatment levels of IL-6 in nonresponders (Pearson correlation coefficient; $\mathrm{r}=-0.68 ; p<.05$ ) (Figure 3A).

Regarding $T N F-\alpha$, both responders and nonresponders showed significantly higher levels of TNF- $\alpha$ production at admission than controls (ANOVA; $p<$ $.01 ; \mathrm{H}=9.73, \mathrm{df}=2$ ) (Dunn's test; $p<.05$ ) (Figures $4 \mathrm{~A}, \mathrm{~B})$. After treatment, the cytokine levels of patients returned to control values (Wilcoxon test; $p<.01$ ) (Figure $4 \mathrm{~A}$ ). However, the decrease reached significance 
Table 2. (A) Number of Lymphocytes and Monocytes with Regard to the Treatment Response. (B) Ratio of Lymphocytes and Monocytes with Regard to the Treatment Response. (C) Levels of C-Reactive Protein (CRP) and the Erythrocyte Sedimentation Rate (ESR).

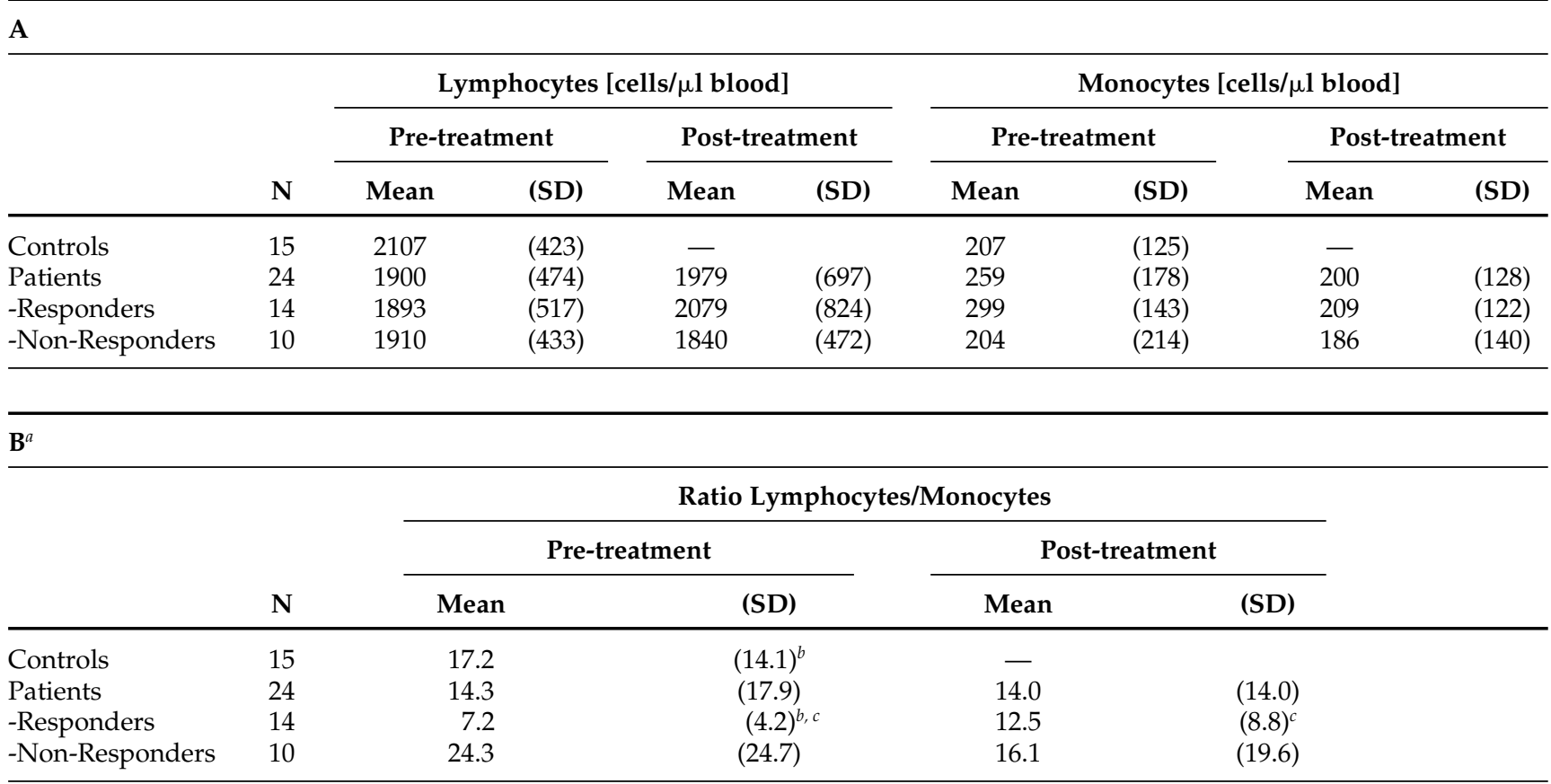

$\mathbf{C}^{j}$

\begin{tabular}{|c|c|c|c|c|c|c|c|c|c|}
\hline & \multirow[b]{3}{*}{$\mathbf{N}$} & \multicolumn{4}{|c|}{ C-reactive Protein [mg/l] } & \multicolumn{4}{|c|}{$\mathrm{ESP}[\mathrm{mm} / \mathrm{h}]$} \\
\hline & & \multicolumn{2}{|c|}{ Pre-treatment } & \multicolumn{2}{|c|}{ Post-treatment } & \multicolumn{2}{|c|}{ Pre-treatment } & \multicolumn{2}{|c|}{ Post-treatment } \\
\hline & & Mean & (SD) & Mean & (SD) & Mean & (SD) & Mean & (SD) \\
\hline Controls & 15 & 1.95 & $(1.40)^{d, h, i}$ & - & & 8.4 & $(2.4)$ & - & \\
\hline Patients & 24 & 5.45 & $(2.59)^{d, e}$ & 2.95 & $(1.84)^{e}$ & 10.2 & $(2.7)$ & 8.8 & $(3.1)$ \\
\hline -Responders & 14 & 5.46 & $(3.26)^{f, h}$ & 3.19 & $(1.89)^{f}$ & 10.2 & $(2.8)$ & 8.5 & (3.3) \\
\hline -Non-Responders & 10 & 5.46 & $(1.32)^{g, i}$ & 2.60 & $(1.80)^{g}$ & 10.2 & $(2.7)$ & 9.3 & (2.9) \\
\hline
\end{tabular}

${ }^{a}$ Kruskal-Wallis-test (Controls, Responders, Non-Responders): $p<.05(\mathrm{H}=6.27, \mathrm{df}=2)$.

$b, h, i p<.05$ (Dunn's test).

$c, e f, g p<.05$ (Wilcoxon test).

${ }^{d} p<.0001$ (Mann-Whitney test).

${ }^{\mathrm{j}}$ Kruskal-Wallis-test (Controls, Responders, Non-Responders: $p<.001(\mathrm{H}=15.21, \mathrm{df}=2)$.

only for the responder subgroup (Wilcoxon test; $p<$ .05) (Figure 4B). In contrast to the data on IL-6, there was no relation between TNF- $\alpha$ levels and monocyte numbers (Table 2A, Figure 2B). No significance of correlation was detected between TNF- $\alpha$ and the degree of clinical severity of MDD (MADRS score) before treatment (Figure 3B).

No effect of the following other variables on the unstimulated secretion of IL- 6 or TNF- $\alpha$ could be found: weight loss, number of depressive episodes, duration of the current episode, and the concentrations of tricyclic antidepressants (data not shown). In addition, we were unable either to detect significant effects of single symptoms of MDD, such as diurnal variation, anxiety, or psychotic features on the production of IL- 6 or TNF- $\alpha$, or to perform a statistical analysis because of the necessity of a larger sample size. On the other hand, insomnia was present in 22 out of 24 patients, not allowing for a reasonable statistical analysis of this factor.

\section{Assessment of Predictive Variables}

To evaluate which of the immunological parameters contributed most to the treatment response as measured by the reduction in psychopathometric scores (predicted variable), a stepwise multiple regression analysis was done. The intercorrelation analysis excluded a relationship among the standard immunological parameters, the amounts of cytokines, and ratios of lymphocyte/monocyte cell numbers. However, further 
Table 3. (A) Correlation Between the Cytokines IL-6 and TNF- $\alpha$ and the Numbers of Lymphocytes and Monocytes. (B) Correlation Between Mononuclear Cells, Cytokines, and the Treatment Response (Reduction in MADRS). (C) Multiple Regression Analysis for the Factors Affecting the Treatment Response (Reduction in MADRS)

A

\begin{tabular}{|c|c|c|c|c|c|c|c|c|}
\hline & \multicolumn{4}{|c|}{ IL-6 } & \multicolumn{4}{|c|}{ TNF- $\alpha$} \\
\hline & \multicolumn{2}{|c|}{ Pre-treatment } & \multicolumn{2}{|c|}{ Post-treatment } & \multicolumn{2}{|c|}{ Pre-treatment } & \multicolumn{2}{|c|}{ Post-treatment } \\
\hline & Monos & Lymphos & Monos & Lymphos & Monos & Lymphos & Monos & Lymphos \\
\hline Controls & -0.18 & -0.28 & - & - & -0.01 & -0.26 & - & - \\
\hline Patients & -0.07 & -0.15 & -0.10 & $-0.47^{a}$ & 0.10 & -0.08 & -0.04 & -0.02 \\
\hline -Responders & 0.09 & -0.49 & -0.30 & $-0.53^{a}$ & 0.30 & -0.14 & 0.18 & -0.03 \\
\hline -Non-Responders & $0.88^{b}$ & 0.05 & 0.22 & -0.40 & 0.05 & 0.17 & -0.18 & -0.07 \\
\hline \multicolumn{9}{|l|}{ B } \\
\hline PMNC & \multirow{2}{*}{\multicolumn{2}{|c|}{$\begin{array}{c}\text { Lymphocytes } \\
0.03\end{array}$}} & Monocytes & IL-6 & TNF- $\alpha$ & \multirow{2}{*}{\multicolumn{3}{|c|}{$\begin{array}{l}\text { Ratio Lymphos/Monos } \\
-0.43^{c}\end{array}$}} \\
\hline-0.04 & & & 0.30 & $-0.62^{c}$ & 0.40 & & & \\
\hline
\end{tabular}

C

Equation: Reduction of MADRS $=77-0.64 \times$ IL- $6-0.465 \times$ Ratio

Multiple $\mathrm{R}=0.772 \quad \mathrm{R}^{2}=0.596 \quad$ adjusted $\mathrm{R}^{2}=0.558 \quad \mathrm{~F}(2.21)=15.5 \quad p=.00007$

Intercorrelation IL-6 versus Ratio : $\mathrm{r}_{\text {IL-Ratio }}=-0.05$

Partial correlations: $\mathrm{r}_{\mathrm{IL}-6}=-0.71 \quad p=.0002 \quad \mathrm{r}_{\text {Ratio }}=-0.59 \quad p=.003$

Abbreviations: Monos = monocytes; Lymphos = lymphocytes.

${ }^{a} p<.05$ (Spearman rank-coefficients).

${ }^{b} p<.01$ (Spearman rank-coefficient).

${ }^{c} p<.01$ (Pearson-coefficient).

examination revealed that the amounts of IL-6/per $10^{5}$ PMNCs and the ratio of lymphocyte/monocyte cell numbers were independent variables with predictive values for the psychopathological response (Table 3C; whereas, the levels of TNF- $\alpha$ and the absolute numbers of mononuclear cell types did not contribute to the clinical outcome in major depressive illness (data not shown).

\section{DISCUSSION}

In a controlled study, we examined different immune parameters, including the cytokines IL- 6 and TNF- $\alpha$, in patients with major depressive disorder with regard to the treatment response. Our results show that IL-6 levels at admission dichotomized patients into subsequent responders and nonresponders; whereas, the unstimulated secretion of TNF- $\alpha$ was uniformly elevated in both patient subgroups at admission and declined according to the psychopathological improvement. After treatment, IL-6 levels were uniformly normalized, regardless of the clinical outcome. We could state that, in our sample, the production of IL-6 seemed to be a predictive marker for the treatment response; whereas, the changes in TNF- $\alpha$ levels paralleled the clinical course. Interestingly, levels of IL-6 showed a high correlation with monocyte counts only in nonresponders, probably indirect evidence that monocytes may be the main source of IL-6 in this subgroup.

Several groups have investigated the interactions between the clinical course of MDD, treatment conditions, and immunological parameters (Frommberger et al. 1997; Maes et al. 1997; McAdams 1993; Seidel et al. 1996; Sluzewska et al. 1995a, 1996). The different scientific and methodological approaches, such as the type of the quantification of the psychopathological response or the effects of psychopharmacological treatments on the immune parameters, led to a variety of results, which, at the moment, are not completely conclusive in regard to the clinical course of the disease. Despite this, the majority of studies suggest that an activation of immunological parameters may, indeed, be relevant for the outcome of depressive illness. To minimize the interfering factors and to analyze the cytokine levels under conditions without major artificial influences, we used a modified whole blood approach with the assessment of the cumulative amounts of cytokines released during the incubation time of the assay. This procedure enables the quantification of cytokine production under conditions fairly similar to the in vivo situation without purification steps or artificial stimulation. Previous studies have shown that this method also correlates with plasma levels of cytokines under physiological conditions (DeGroote et al. 1993).

Regarding the production of the cytokine IL-6 under 
A

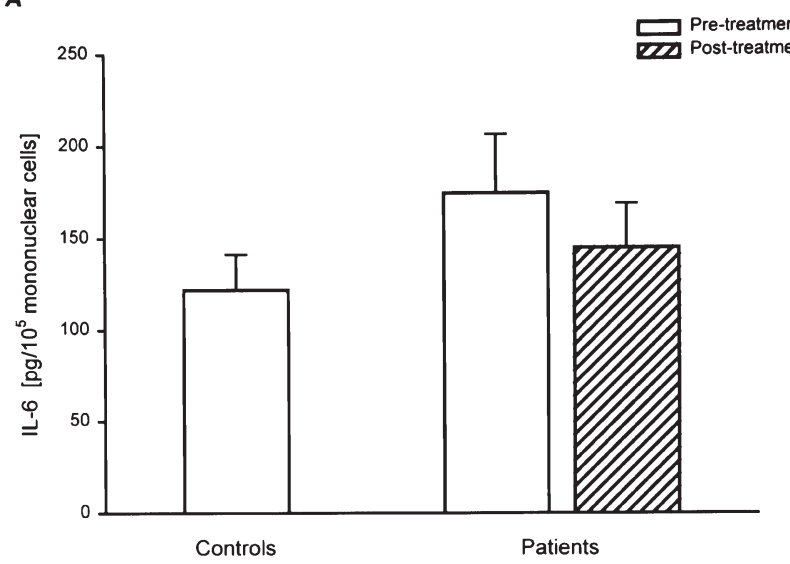

B

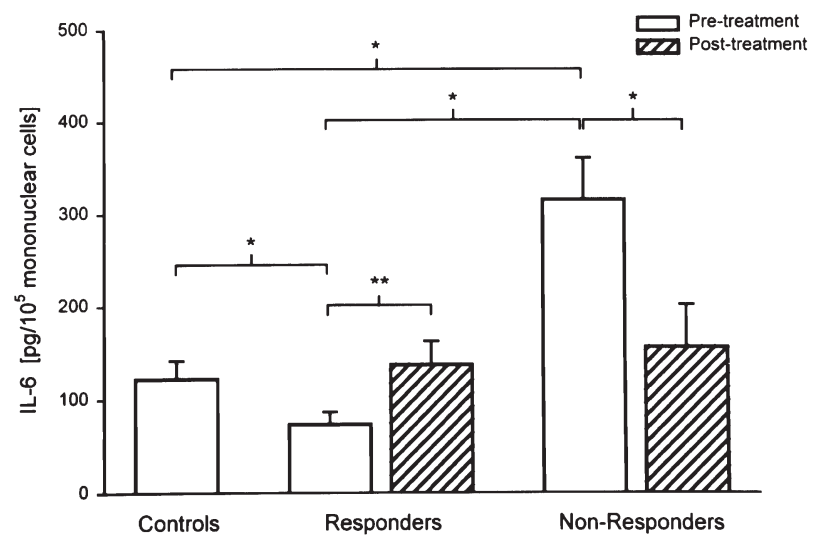

Figure 1. Production of IL-6 (pg/10 5 mononuclear cells) in whole blood under baseline conditions with regard to all patients and controls (A) [Mann-Whitney test; $p=.95, \mathrm{NS}$ ] and with regard to responders and nonresponders; (B) after 6 weeks of antidepressive treatment [(for comparisons between subgroups: ANOVA; $p<.0001 ; \mathrm{H}=16.9$; $\mathrm{df}=2$; Dunn's test; ${ }^{*} p<.05$ ) (for repeated measures: Wilcoxon test; ${ }^{* *} p<.01$ for responders, ${ }^{*} p=.05$ for nonresponders)].

our conditions, the pre- and post-treatment values of the total group of our patients did not differ from those of the control subjects. However, if looking retrospectively at patient subgroups according to their clinical outcome, responders produced significantly less and nonresponders significantly more IL-6 at admission than controls. In both groups, the normalization of IL-6 levels occurred under the treatment condition, regardless of the clinical outcome. Interestingly, higher levels of IL-6 at admission were closely correlated with a subsequent insufficient psychopathological treatment response and yielded a predictive variable in multiple regression analysis. In accordance with other results (Frommberger et al. 1997; Maes et al. 1997; Sluzewska et al. 1995a), these data underscore the relevance of IL-6
A
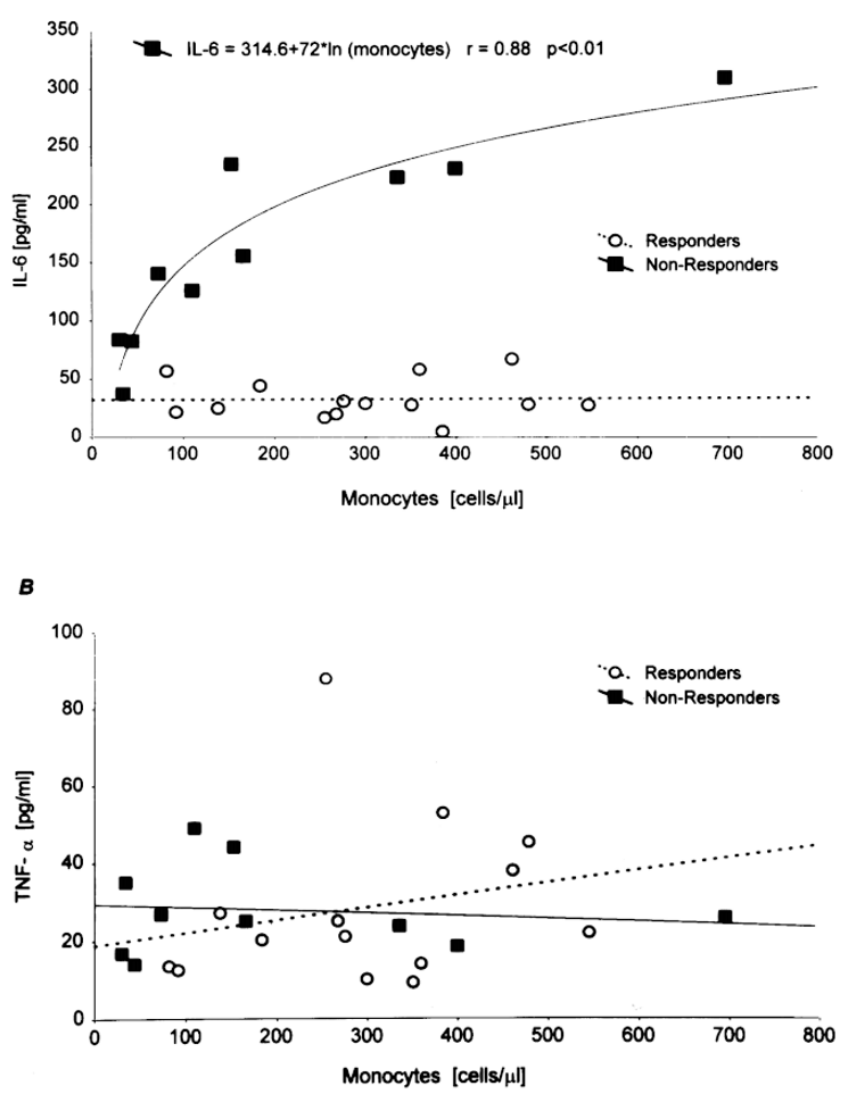

Figure 2. Correlation between IL-6 (A) or TNF- $\alpha$ (B) (pg/ $\mathrm{ml})$ and the number of monocytes.

and its soluble receptor (sIL-6R) for the treatment response. Increased pretreatment production of IL-6 in subsequent nonresponders coincides well with the results of Sluzewska et al. (1995a) and Maes et al. (1997), who found elevated plasma levels in patients refractory to antidepressive treatment. On the other hand, our post-treatment finding of a uniform normalization of IL-6 in both patient subgroups after treatment differs from the findings of Maes et al. (1995a; 1997), who did not observe changes of this cytokine after remission. The reasons for this difference are not yet clear; for example, variations in the patient samples, study methodology, and treatment modalities may account for our differing results in this respect.

Pretreatment levels of TNF- $\alpha$ were not correlated with psychopathological improvement and, therefore, without predictive value, because the initial levels were increased in both patient subgroups in comparison to the healthy controls. For the first time, these data support an increase in the production of TNF- $\alpha$ in an acute state of depression, which was related to the time of hospital admission. A variety of factors, such as IL-1 $\beta$ and interferon- $\gamma$ are able to induce the production of $\mathrm{TNF}-\alpha$. Interestingly, a secretion of $\mathrm{TNF}-\alpha$ is even de- 
A

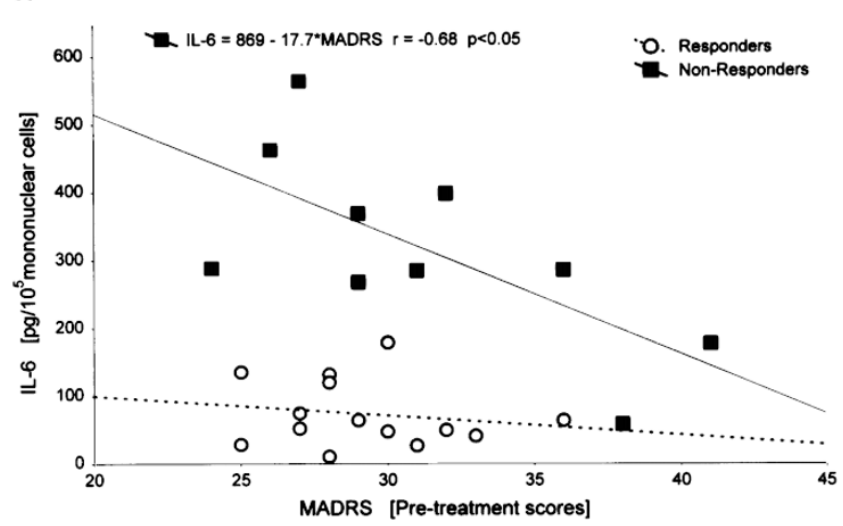

B

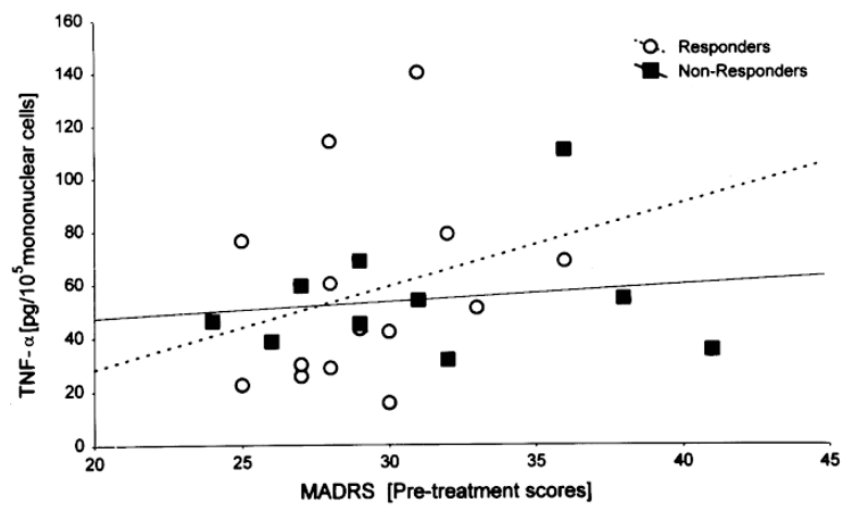

Figure 3. Correlation between IL- 6 (A) or TNF- $\alpha$ (B) (pg/ $10^{5}$ mononuclear cells) and the degree of clinical severity of MDD (MADRS score) before treatment.

tectable in humans under conditions of subclinical stimulation with low amounts of bacteria-derived lipopolysaccharide (Vedder et al. 1999), pointing to the possible relevance of this type of infection for the induction of TNF- $\alpha$ in MDD. Such subclinical infections may then result in increased production of this cytokine contributing to clinical symptomatology in MDD. We could further speculate that changes in the cytokines, such as those shown by our study for TNF- $\alpha$ and IL-6, may then affect neurotransmitter systems in the CNS, leading to psychopathological changes and subsequently affecting the treatment response in MDD. The decrease of TNF- $\alpha$ levels under treatment in the responder subgroup favors the hypothesis of an effective down-regulation of TNF- $\alpha$ by tricyclic antidepressants, which may clinically confirm in vitro results of the cytokine-modulating effects of antidepressants in human mononuclear cells (Xia et al. 1996).

In our patients, IL-6 levels below normal values at admission apparently heralded a treatment response; whereas, increased levels were associated with a less favorable outcome. Therefore, we can speculate that the up-regulated secretion dynamics might be related to
A

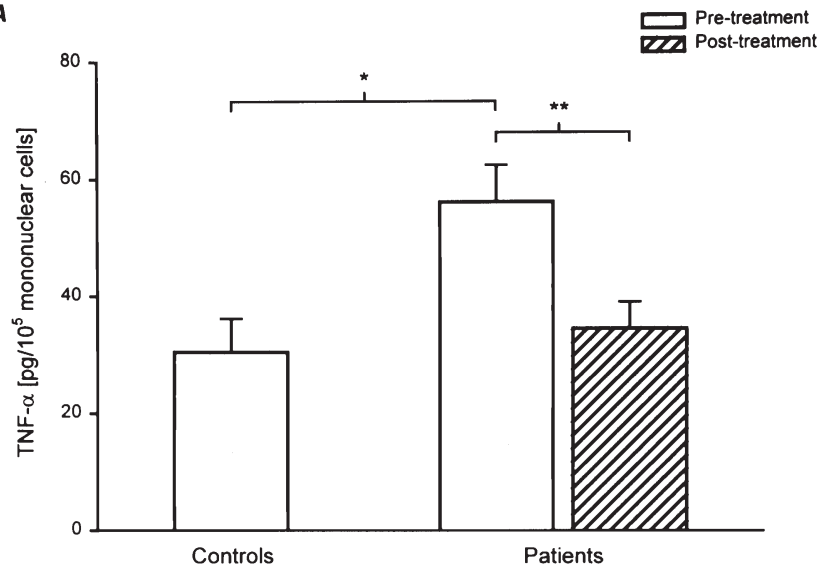

B
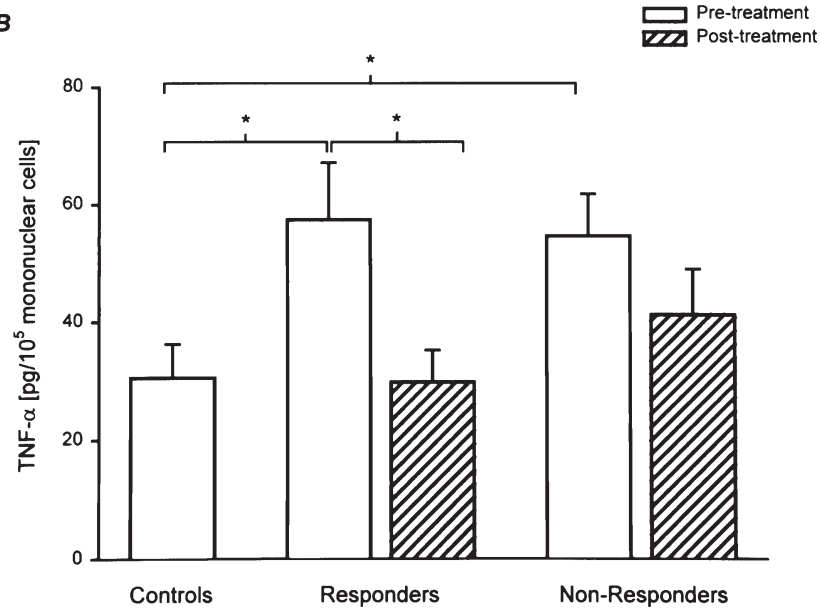

Figure 4. Production of TNF- $\alpha$ (pg/10 5 mononuclear cells) in whole blood under baseline conditions with regard to all patients and controls (A) [Mann-Whitney test; ${ }^{*} p<.05$; Wilcoxon test; ${ }^{* *} p<.01$ ] and with regard to responders and nonresponders (B) after 6 weeks of antidepressive treatment [(for comparisons between subgroups: ANOVA; $p<.01 ; \mathrm{H}=$ 9.73; df = 2; Dunn's test; ${ }^{*} p<.05$ ) (for repeated measures: Wilcoxon test; ${ }^{*} p<.05$ for responders)].

only "hesitant" remission or even treatment-refractory types of MDD. Alternatively, the remitting subgroup with the low IL-6 production might have already passed a state of increased secretion; whereas, the nonresponding group would still be in the "early" state of immunological activation. This assumption is also supported by the significantly elevated production of the cytokine TNF- $\alpha$ in both patient subgroups at admission, with only the subgroup of responders returning to control levels. Although not significant, nonresponders still showed an increased production of TNF- $\alpha$ after the 6-week period, pointing to a possible persistence of the immune activation.

Regarding the treatment response under our condi- 
tions, the ratio of lymphocyte/monocyte counts proved to be the only variable, besides IL-6, which independently contributed to the prediction of the outcome when introduced into the multiple regression model. Interestingly, mononuclear cell counts, apart from a trend to increased pretreatment values in the responders, contributed little to the prediction of the clinical outcome under our conditions. Results from the groups of Maes et al. (1992; 1997) and Seidel et al. (1996) show an increased number of monocytes and possibly also alterations of monocyte functions in the course of MDD. Taken together, these data point to the importance of both functional and numerical changes of the immune system in MDD.

In conclusion, we show here that, in patients with an MDD, the treatment response to an antidepressant is related to a differential pattern of immune activation including production of the cytokines IL- 6 and TNF- $\alpha$. Our results enforce the view that immune processes may be significantly involved in the treatment response in affective disorder. Moreover, the unstimulated production of IL-6 in mononuclear cells at admission might even enable the prospective description of the type of the psychopathological response after a treatment interval of several weeks. Whether the results, which we have obtained under a medication with amitriptyline, are also transferable to treatment conditions with other classes of antidepressants, such as the selective serotonine reuptake inhibitors, must be shown in future studies.

\section{ACKNOWLEDGMENTS}

We thank Mrs. C. Salmonson for her support with the final version of the manuscript, Mrs. A. Tittmar for her secretarial assistance, and D. Fischer-Barnicol for his considerable help with the acquisition of samples. The study was supported by grants from the "Deutsche Forschungsgemeinschaft" (SFB 297, Projekt A 6) and the "P. E. Kempkes Stiftung."

\section{REFERENCES}

American Psychiatric Association (1994): Diagnostic and Statistical Manual of Mental Disorders IV (DSM IV). Washington, DC, the American Psychiatric Association

Berk M, Wadee AA, Kuschke RH, O'Neill-Kerr A (1997): Acute phase proteins in major depression. J Psychosom Res 43:529-534

Brambilla F, Maggioni M (1998): Blood levels of cytokines in elderly patients with major depressive disorder. Acta Psychiat Scand 97:309-313

Connor TJ, Leonard BE (1998): Depression, stress, and immunological activation: The role of cytokines in depressive disorders. Life Sci 62:583-606

Connor TJ, Song C, Leonard BE, Merali Z, Anisman H (1998): An assessment of the effects of central interleu- kin- $1 \beta,-2,-6$, and tumor necrosis factor- $\alpha$ administration on some behavioral, neurochemical, endocrine, and immune parameters in the rat. Neuroscience 84:923-933

De Groote D, Gevaert Y, Lopez M, Gathy R, Fauchet F, Dehart I, Jadoul M, Radoux D, Franchimont P (1993): Novel method for the measurement of cytokine production by a one-stage procedure. J Immunol Methods 163:259-267

Frommberger UH, Bauer J, Haselbauer P, Raulin A, Riemann D, Berger M (1997): Interleukin-6-(IL-6) plasma levels in depression and schizophrenia: Comparison between the acute state and after remission. Eur Arch Psychiat Clin Neurosci 247:228-233

Hamilton M (1960): A rating scale for depression. J Neurol Neurosurg Psychiat 23:56-62

Maes M (1995): Evidence for an immune response in major depression: A review and hypothesis. Prog Neuro-Psychopharmacol \& Biol Psychiat 19:11-38

Maes M, Bosman E, Suy E, Vandervorst C, De Jonckheere C, Raus J (1991): Depression-related disturbances in mitogen-induced lymphocyte responses and interleukin-1 $\beta$ and soluble interleukin-2 receptor production. Acta Psychiat Scand 84:379-386

Maes M, van der Planken M, Stevens WH (1992): Leucocytosis, monocytosis, and neutrophilia: Hallmarks of severe depression. J Psychiat Res 26:125-134

Maes M, Bosmans E, Meltzer HY, Scharpe S, Suy E (1993): Interleukin-1 $\beta$ : A putative mediator of HPA axis hyperactivity in major depression? Am J Psychiat 150:1189-1193

Maes M, Scharpe S, Meltzer HY, Okayli G, Bosmans E, D'Hondt P, Vanden Bossche V, Cosyns P (1994): Increased neopterin and interferon- $\gamma$ secretion and lower availability of L-tryptophan in major depression: Further evidence for an immune response. Psychiat Res 54:143-160

Maes M, Vandoolaeghe E, Ranjan R, Bosmans E, Bergmans $R$, Desnyder A (1995a): Increased serum interleukin-1receptor-antagonist concentrations in major depression. J Affect Disord 36:2-36

Maes M, Meltzer HY, Bosmans E, Bergmans R, Vandoolaeghe E, Ranjan R, Desnyder R (1995b): Increased plasma concentrations of interleukin-6, soluble interleukin-6, soluble interleukin-2, and transferrin receptor in major depression. J Affect Disord 34:301-309

Maes M, Bosmans E, De Jongh R, Kenis G, Vandoolaeghe E, Neels H (1997): Increased serum IL-6 and IL-1 receptor antagonist concentrations in major depression and treatment-resistant depression. Cytokine 9:853-858

McAdams, LBE (1993): Neutrophil and monocyte phagocytosis in depressed patients. Prog Neuropsychopharmacol Biol Psychiat 17:971-984

Montgomery SA, Asberg M (1979): A new depression scale designed to be sensitive to change. Br J Psychiat 134:382-389

Müller N, Ackenheil M (1998): Psychoneuroimmunology and the cytokine action in the CNS: Implications for psychiatric disorders. Prog Neuro-Psychopharmacol \& Biol Psychiat 22:1-33

Ravindran AV, Griffiths J, Merali Z, Anisman H (1998): Circulating lymphocyte subsets in major depression and 
dysthymia with typical or atypical features. Psychosom Med 60:283-289

Seidel A, Arold V, Hunstiger M, Rink L, Behnisch A, Kirchner $H$ (1995): Cytokine production and serum proteins in depression. Scand J Immunol 41:534-538

Seidel A, Arolt V, Hunstiger M, Rink L, Behnisch A, Kirchner $H$ (1996): Major depressive disorder is associated with elevated monocyte counts. Acta Psychiat Scand 94:198-204

Sluzewska A, Rybakowski J, Bosmans E, Sobieska M, Berghmans R, Maes M, Wiktorowicz K (1996): Indicators of immune activation in major depression. Psychiat Res 64:161-167

Sluzewska A, Rybakowski JK, Laciak M, Mackiewicz A, Sobieska M (1995a): Interleukin-6 serum levels in depressed patients before and after treatment with fluoxetine. Ann NY Acad Sci 762:474-476

Sluzewska A, Rybakowski JK, Sobieska M, Bosmans E, Pol- let H, Wiktorowicz K (1995b): Increased levels of $\alpha-1$ acidic glycoprotein and interleukin-6 in refractory depression. Depression 3:170-175

Vedder H, Schreiber W, Yassouridis A, Gudewill S, Galanos C, Pollmächer T (1999): Dose-dependence of bacterial lipopolysaccharide (LPS) effects on the peak response and time course of the immune-endocrine host response in humans. Inflamm Res 48:67-74

Weizman R, Laor N, Podliszewski E, Notti I, Djaldetti M, Bessler H (1994): Cytokine production in major depressed patients before and after clomipramine treatment. Biol Psychiat 35:42-47

Wittchen HU, Zaudig M, Fydrich, T (1995): Strukturiertes Klinisches Interview für DSM-IV (SKID-I und SKID-II). Göttingen, Hogrefe Verlag

Xia Z, DePierre JW, Nassberger L (1996): Tricyclic antidepressants inhibit IL-6, IL-1 $\beta$ and TNF- $\alpha$ release in human blood monocytes and IL-2 and interferon- $\gamma$ in T cells. Immunopharmacology 34:27-37 